\title{
Fast Sub-aperture Stitching Algorithm Using Partial Derivatives
}

\author{
Yiwei Chen ${ }^{1,2 *}$, Erlong Miao ${ }^{1}$, Yongxin Sui ${ }^{1}$, and Huaijiang Yang $^{1}$ \\ ${ }^{1}$ State Key Laboratory of Applied Optics, Changchun Institute of Optics, Fine Mechanics and Physics, \\ Chinese Academy of Sciences, Changchun, Jilin 130033, China \\ ${ }^{2}$ University of Chinese Academy of Sciences, Beijing 100049, China
}

(Received October 6, 2014 : revised December 4, 2014 : accepted December 8, 2014)

\begin{abstract}
For large optical elements which are tested by many sub-apertures, it takes too much time for a sub-aperture stitching algorithm to get the stitching result. To solve this problem, we propose a fast sub-aperture stitching algorithm to quickly compensate for piston, tilt, and defocus errors. Moreover, the new algorithm is easy to understand and program. We use partial derivatives of measurement data to separately solve piston, tilt, and defocus errors. First, we show that the new algorithm has a lower time complexity than the currently used algorithm. Although simulation results indicate that the accuracy of the new algorithm is lower than the current algorithm in all 20 simulations, our experimental results validate the algorithm and show it is sufficiently accurate for general use.
\end{abstract}

Keywords : Optical testing, Interferometer, Large optical elements, Sub-aperture stitching algorithm OCIS codes : (220.4840) Optical testing; (120.3180) Interferometry; (120.6650) Surface measurements, figure; (120.4630) Optical inspection

\section{INTRODUCTION}

With the development of astronomical optics, space optics [1-3] and inertial confinement fusion, large-aperture optical systems have been widely used. For very large optical elements, however, the aperture may be too large for the interferometer to test in one pass. In 1982, C. J. Kim solved this problem with the sub-aperture stitching method [4]. However, location errors are inevitable with this method. Thus, researchers use overlapping areas between the sub-apertures to compensate for location errors [5-9] such as piston, tilt, defocus, clocking, and position errors. In most cases, only piston, tilt, and defocus errors are corrected. For large optical elements which are tested by many sub-apertures, it takes much time for a sub-aperture stitching algorithm to get the stitching result. To quickly compensate for piston, tilt, and defocus errors, we develop a fast algorithm, which is easy to understand and program.

In Section 2, we briefly introduce our algorithm. In Section 3, we compare the time complexity of our algorithm with the current algorithm. In Section 4, we study the accuracy of our algorithm using simulation. In Section 5, we discuss an experimental result demonstrating the effecti- veness of the proposed algorithm. Finally, we present our conclusions in Section 6.

\section{FAST ALGORITHM FOR SUB-APERTURE STITCHING}

The current algorithm uses a least-squares method to remove relative piston, tilt and defocus errors directly. In our algorithm, we calculate the partial derivative of data before using a least-squares method to remove relative piston, tilt and defocus errors. The reason for calculating the partial derivative of data is that it makes the least-squares method much easier. Actually, using the least-squares method after calculating the partial derivative of data is just averaging, so it makes the algorithm faster than current algorithm.

For convenience, we only discuss the testing of spherical optical elements in this article; however, with some modifications, this algorithm can be easily applied to the testing of both flat and aspheric optical elements.

One sub-aperture, usually the center sub-aperture, is selected as the reference sub-aperture. The remaining

\footnotetext{
*Corresponding author: cyw198788@163.com

Color versions of one or more of the figures in this paper are available online.
} 
sub-apertures have nonzero piston, tilt, and defocus errors that are relative to the reference sub-aperture. Thus, we compensate for relative piston, tilt and defocus errors [9] as follows:

$$
z_{i}^{*}(x, y)=z_{i}(x, y)+a_{i} x+b_{i} y+c_{i}+d_{i}\left(x^{2}+y^{2}\right)
$$

where $x$ and $y$ are the coordinates of pixels; $\mathrm{z}_{\mathrm{i}}(x, y)$ is the testing value measured for the $\mathrm{i}$-th sub-aperture; $z_{i}^{*}(x, y)$ is the value after compensation; $a_{\mathrm{i}}$ and $b_{i}$ are the coefficients of the relative tilt of the $\mathrm{i}$-th sub-aperture in the $x$ and $y$ directions, respectively, $c_{i}$ is the coefficient of the relative piston value of the i-th sub-aperture, and $d_{i}$ is coefficient of relative defocus.

Assuming that there are $\mathrm{N}$ sub-apertures and that the reference sub-aperture is number 1 , current sub-aperture stitching algorithms use the least-squares method to directly calculate the necessary compensations [5-9]:

$$
\begin{aligned}
\min & =\sum_{i=2} \sum_{j=i . . N}^{j \cap i}\left(\left(z_{i}(x, y)+a_{i} x+b_{i} y+c_{i}+d_{i}\left(x^{2}+y^{2}\right)\right)\right. \\
& \left.-\left(z_{j}(x, y)+a_{j} x+b_{j} y+c_{j}+d_{j}\left(x^{2}+y^{2}\right)\right)\right)^{2} \\
& +\sum_{k=2 \ldots N}^{k-1}\left(\left(z_{k}(x, y)+a_{k} x+b_{k} y+c_{k}+d_{k}\left(x^{2}+y^{2}\right)\right)-z_{1}(x, y)\right)^{2}
\end{aligned}
$$

In our algorithm, we first calculate the second-order partial derivative of Equation 1 with respect to the independent variable $\mathrm{x}$ :

$$
\frac{\partial^{2} z_{i}^{*}(x, y)}{\partial x^{2}}=\frac{\partial^{2} z_{i}(x, y)}{\partial x^{2}}+2 d_{i}
$$

Then, we use the least-squares method to calculate the needed compensation between two adjacent sub-apertures:

$$
\begin{array}{ll}
\min =\sum^{i n_{j}}\left(\frac{\partial^{2}\left(z_{i}(x, y)-z_{j}(x, y)\right)}{\partial x^{2}}+2\left(d_{i}-d_{j}\right)\right)^{2} & i, j \neq 1 \\
\min =\sum^{k n !}\left(\frac{\left(\partial^{2}\left(z_{k}(x, y)-z_{1}(x, y)\right)\right.}{\partial x^{2}}+2 d_{k}\right)^{2} & k \neq 1
\end{array}
$$

Solving Equation 4, we obtain the following over-determined linear equations:

$$
\begin{array}{ll}
d_{i}-d_{j}=\frac{1}{2 N_{i j}} \sum^{i \cap j} \frac{\partial^{2}\left(z_{j}(x, y)-z_{i}(x, y)\right)}{\partial x^{2}} & i, j \neq 1 \\
d_{k}=\frac{1}{2 N_{k}} \sum^{k \cap 1} \frac{\partial^{2}\left(z_{1}(x, y)-z_{k}(x, y)\right)}{\partial x^{2}} & k \neq 1
\end{array}
$$

As we can see, using a least-squares method after calculating the partial derivative of data is just averaging. Here, $\mathrm{N}_{\mathrm{ij}}$ is the total number of pixels in the overlapping area of the $\mathrm{i}$-th sub-aperture and the $\mathrm{j}$-th sub-aperture, and $\mathrm{N}_{\mathrm{k}}$ is the total number of pixels in the overlapping area of 1-st sub-aperture and k-th sub-aperture.

We can easily use the weighted least-squares method to solve the over-determined linear equations by setting the weight equal to the total number of pixels in the corresponding overlapping area.

Using the calculated coefficients of the relative defocus, we compensate the defocus errors:

$$
f_{i}(x, y)=z_{i}(x, y)+d_{i}\left(x^{2}+y^{2}\right)
$$

where $f_{i}(x, y)$ is the data of the i-th sub-aperture after compensating for the defocus error. According to Equation 2 and Equation 6, we obtain

$$
z_{i}^{*}(x, y)=f_{i}(x, y)+a_{i} x+b_{i} y+c_{i}
$$

To compensate for the relative tilt errors, we calculate the first-order partial derivative of Equation 7 with respect to independent variable $\mathrm{x}$ and the independent variable $\mathrm{y}$ :

$$
\begin{aligned}
& \frac{\partial z_{i}^{*}(x, y)}{\partial x}=\frac{\partial f_{i}(x, y)}{\partial x}+a_{i} \\
& \frac{\partial z_{i}^{*}(x, y)}{\partial y}=\frac{\partial f_{i}(x, y)}{\partial y}+b_{i}
\end{aligned}
$$

We use a similar procedure to calculate $a_{i}$ and $b_{i}$. Then, we compensate for the tilt errors:

$$
z_{i}^{*}(x, y)=g_{i}(x, y)+c_{i}
$$

where $g_{i}(x, y)$ is the data of $\mathrm{i}$-th sub-aperture after compensating for the tilt and defocus errors.

Finally, we use the same method to compensate for the piston errors and stitch the resulting measurement data to obtain a full-aperture result.

\section{TIME COMPLEXITY OF THE ALGORITHM}

In this section, we assume that there are only two sub-apertures, where one sub-aperture is the reference subaperture, and the overlapping area is a square $(n \times n$ pixels).

In the algorithm currently in use, Equation 2 leads to linear equations:

$$
\left[\begin{array}{c}
\sum x \Delta(x, y) \\
\sum y \Delta(x, y) \\
\sum \Delta(x, y) \\
\sum\left(x^{2}+y^{2}\right) \Delta(x, y)
\end{array}\right]=\left[\begin{array}{cccc}
\sum x^{2} & \sum x y & \sum x & \sum x\left(x^{2}+y^{2}\right) \\
\sum x y & \sum y^{2} & \sum y & \sum y\left(x^{2}+y^{2}\right) \\
\sum x & \sum y & n \times n & \sum\left(x^{2}+y^{2}\right) \\
\sum x\left(x^{2}+y^{2}\right) & \sum\left(x^{2}+y^{2}\right) & \sum y\left(x^{2}+y^{2}\right) & \sum\left(x^{2}+y^{2}\right)^{2}
\end{array}\right]\left[\begin{array}{l}
a \\
b \\
c \\
d
\end{array}\right]
$$


TABLE 1 . Time complexity

\begin{tabular}{c|c|c}
\hline \hline & Current algorithm & New algorithm \\
\hline Multiplication operations & $12 n^{2}$ & $5 n^{2}$ \\
\hline Addition operations & $18 n^{2}$ & $12 n^{2}$ \\
\hline
\end{tabular}

where $\Delta(x, y)$ is the disparity between the two measurements $\left(z_{1}(x, y)-z_{2}(x, y)\right)$.

Solving Equation 11, we obtain the coefficients $a, b, c$, and $d$, and we can use Equation 1 to compensate for the errors. In the current algorithm, we use about $12 n^{2}$ multiplication operations and about $18 n^{2}$ addition operations (shown in Table 1).

In our new algorithm, we use differences to calculate partial derivatives. For instance, we find the first-order partial derivative using

$$
\frac{\partial f_{i}(x, y)}{\partial x}=\frac{\left(f_{i}(x+\Delta x, y)-f_{i}(x, y)\right)+\left(f_{i}(x, y)-f_{i}(x-\Delta x, y)\right)}{2 \Delta x}
$$

where $\Delta x$ is the distance between two adjacent pixels. In our method, we do not divide $\left(f_{i}(x+\Delta x, y)-f_{i}(x, y)\right)+$ $\left.f_{i}(x, y)-f_{i}(x-\Delta x, y)\right)$ by $2 \Delta x$ for each point. For instance, when we obtain

$$
a_{k}=\frac{1}{N_{k}} \sum^{k \cap 1} \frac{\partial\left(f_{1}(x, y)-f_{k}(x, y)\right)}{\partial x}
$$

we calculate it using

$$
a_{k}=\frac{1}{2 \Delta x} \frac{1}{N_{k}} \sum^{k \cap 1}\left(f_{i}(x+\Delta x, y)-f_{i}(x, y)\right)+\left(f_{i}(x, y)-f_{i}(x-\Delta x, y)\right)
$$

Thus, we use about $5 n^{2}$ multiplication operations and about $12 n^{2}$ addition operations in our algorithm (shown in Table 1).

In Table 1, we can see that our algorithm has a much lower time complexity than the current algorithm.

\section{SIMULATION}

In the simulation, a measurement result $(501 \times 801$ pixels, shown in Fig. 1) was first divided into two sub-apertures. After dividing the measurement result into sub-apertures, we add random noise ([-0.001, 0.001] wavelength) and piston, tilt, and defocus errors to each sub-aperture. We use both the current algorithm and the new algorithm to calculate the compensators. We repeated the simulation calculations 20 times. In all 20 simulations, the accuracy of the new algorithm is lower than that of the current algorithm. We present one typical result in Table 2 .

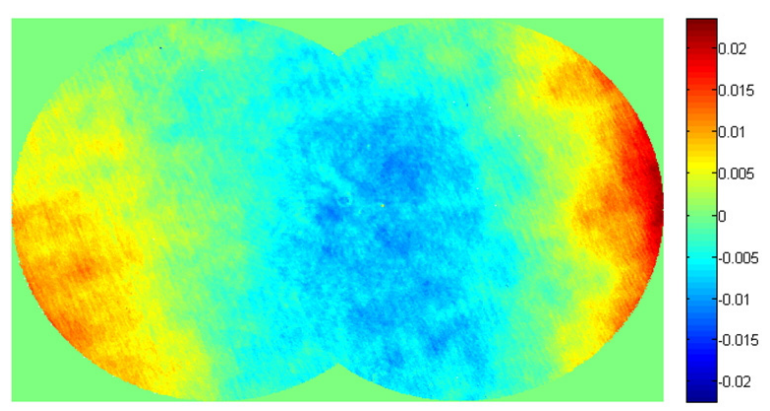

FIG. 1. Measurement result used for the simulation. All units are in wavelengths.

TABLE 2. Simulation results

\begin{tabular}{c|c|c|c}
\hline \hline & $\begin{array}{c}\mathrm{a} \\
\left(10^{-5} \text { wavelength/ }\right. \\
\text { pixel })\end{array}$ & $\begin{array}{c}\mathrm{b} \\
\left(10^{-5} \text { wavelength/ }\right. \\
\text { pixel })\end{array}$ & $\begin{array}{c}\mathrm{d} \\
\left(10^{-5} \text { wavelength/ }_{\text {pixel }^{2}}\right)\end{array}$ \\
\hline $\begin{array}{c}\text { Initial } \\
\text { Error }\end{array}$ & 3.6153 & 2.3282 & -2.2228 \\
\hline $\begin{array}{c}\text { Old } \\
\text { algorithm }\end{array}$ & 3.6167 & 2.3276 & -2.2228 \\
\hline $\begin{array}{c}\text { New } \\
\text { algorithm }\end{array}$ & 3.6189 & 2.3154 & -2.2223 \\
\hline
\end{tabular}

\section{EXPERIMENT}

In this section, we test a convex spherical surface (caliber of $100 \mathrm{~mm}$ and radius of $76.56 \mathrm{~mm}$ ) using a sub-aperture stitching interferometer (a Fizeau interferometer whose working wavelength is $632.8 \mathrm{~nm}$ ) with a reference surface whose F-number is 1.5. A real system including a real sample is shown in Fig. 2. The layout of the sub-apertures is shown in Fig. 3. The measurement results using our algorithm are shown in Fig. 4(a). Comparing with the measurement results obtained using a full-aperture interferometer (shown in Fig. 4(b)), the root mean squared error was $0.003014 \lambda$ and the residual error image is shown in Fig. 5.

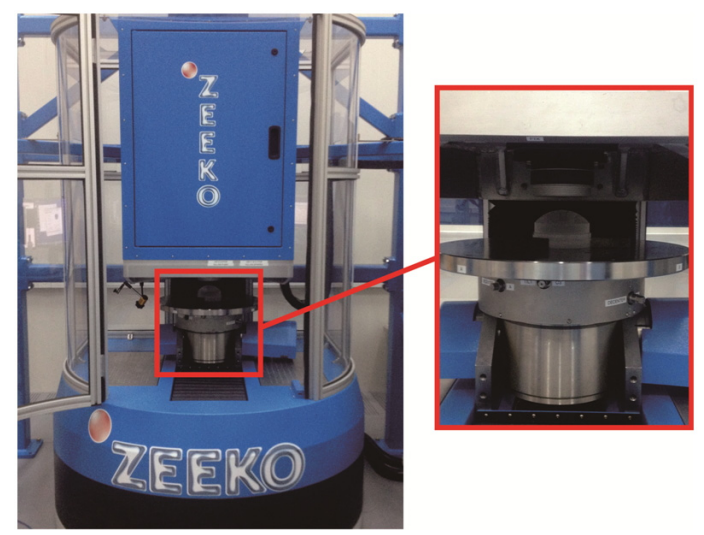

FIG. 2. Real system including real sample. 


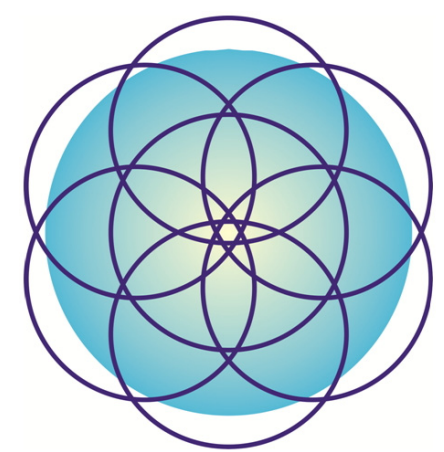

FIG. 3. Layout of the sub-apertures.

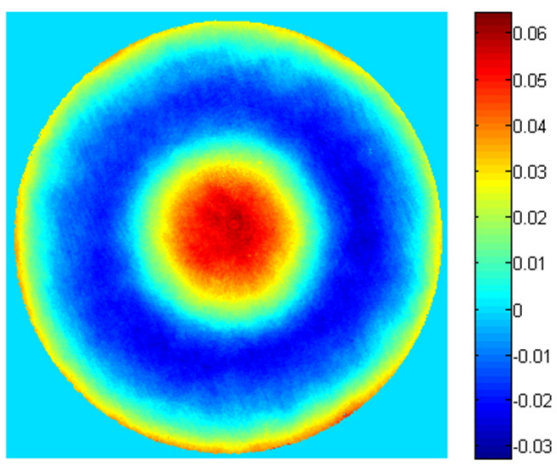

(a)

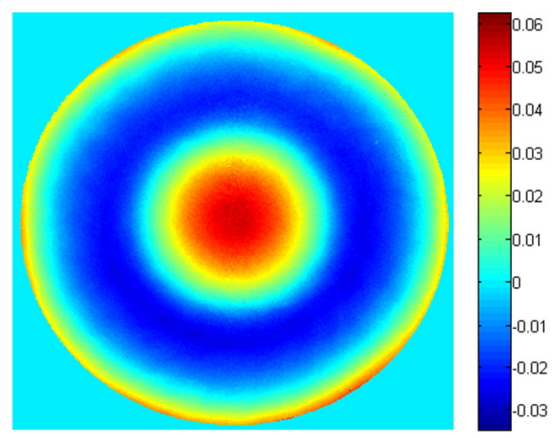

(b)

Unit: micron

FIG. 4. Experimental results: (a) stitched using our algorithm; (b) full-aperture measurement.

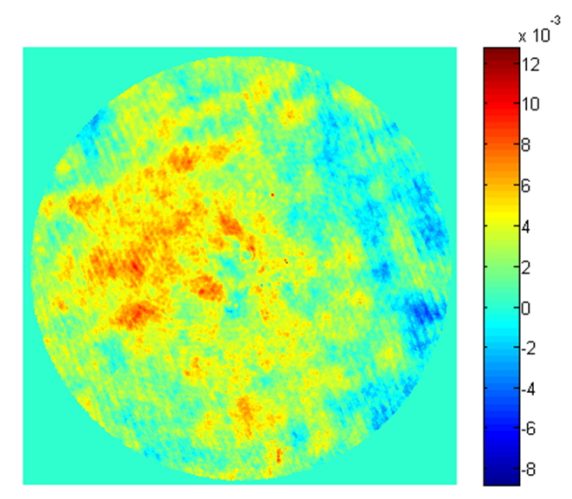

Unit: micron

FIG. 5. Residual error image.

\section{CONCLUSION}

We propose a fast algorithm for sub-aperture stitching to quickly compensate for piston, tilt, and defocus errors. We prove that our algorithm runs more than two times faster than the current algorithm. Although the simulation results demonstrate that the accuracy of the new algorithm is lower than that of the current algorithm in all 20 simulations, the new algorithm is sufficiently accurate for generally use. Using an experimental measurement, we verified the validity of the new algorithm and demonstrated successful sub-aperture stitching.

\section{REFERENCES}

1. X. L. Li, M. Xu, X. D. Ren, and Y. T. Pei, “An optical design of off-axis four-mirror-anastigmatic telescope for remote sensing," J. Opt. Soc. Korea 16, 243-246 (2012).

2. X. L. Li, M. Xu, and Y. T. Pei, "Optical design of an off-axis five-mirror-anastigmatic telescope for near infrared remote sensing," J. Opt. Soc. Korea 16, 343-348 (2012).

3. H. Jin, J. Lim, Y. Kim, and S. Kim, "Optical design of a reflecting telescope for cubesat," J. Opt. Soc. Korea 17, 533-537 (2013).

4. C. J. Kim, "Polynomial fit of interferograms," Appl. Opt. 21, 4521-4525 (1982).

5. M. Otsubo, K. Okada, and J. Tsujiuchi, "Measurement of large plane surface shape with interferometric aperture synthesis," Proc. SPIE 1720, $444-447$ (1992).

6. M. Otsubo, K. Okada, and J. Tsujiuchi, "Measurement of large plane surface shapes by connecting small-aperture," Opt. Eng. 33, 608-613 (1994).

7. M. Sjödahl and B. F.Oreb, "Stitching interferometric measurement data for inspection of large optical components," Opt. Eng. 41, 403-408 (2002).

8. D. Golini, G. W. Forbes, and P. E. Murphy, "Method for self-calibrated subaperture stitching for surface figure measurement," U.S. Patent:6956657B (2005).

9. J. Fleig, P. Dumas, P. E. Murphy, and G. W. Forbes, "An automated subaperture stitching interferometer workstation for spherical and aspherical surfaces," Optical Science and Technology, SPIE's 48th Annual Meeting. International Society for Optics and Photonics, 296-307 (2003). 